\title{
Unhindered Brownian Motion of Individual Nanoparticles in Liquid-Phase Scanning Transmission Electron Microscopy
}

Yesibolati, Murat Nulati; Mortensen, Kim I.; Sun, Hongyu; Brostrøm, Anders; Tidemand-Lichtenberg, Sofie; Mølhave, Kristian

Published in:

Nano Letters

Link to article, DOI:

10.1021/acs.nanolett.0c02352

Publication date:

2020

Document Version

Peer reviewed version

Link back to DTU Orbit

Citation (APA):

Yesibolati, M. N., Mortensen, K. I., Sun, H., Brostrøm, A., Tidemand-Lichtenberg, S., \& Mølhave, K. (2020). Unhindered Brownian Motion of Individual Nanoparticles in Liquid-Phase Scanning Transmission Electron Microscopy. Nano Letters, 20(10), 7108-7115. https://doi.org/10.1021/acs.nanolett.0c02352

\section{General rights}

Copyright and moral rights for the publications made accessible in the public portal are retained by the authors and/or other copyright owners and it is a condition of accessing publications that users recognise and abide by the legal requirements associated with these rights.

- Users may download and print one copy of any publication from the public portal for the purpose of private study or research.

- You may not further distribute the material or use it for any profit-making activity or commercial gain

- You may freely distribute the URL identifying the publication in the public portal 


\section{Unhindered Brownian Motion of Individual Nanoparticles in Liquid Phase Scanning Transmission Electron Microscopy}

Murat Nulati Yesibolati, Kim I. Mortensen, HONGYU SUN, Anders

Brostrøm, Sofie Tidemand-Lichtenberg, and Kristian Mølhave

Nano Lett., Just Accepted Manuscript • DOI: 10.1021/acs.nanolett.0c02352 • Publication Date (Web): 17 Jul 2020

Downloaded from pubs.acs.org on July 20, 2020

\section{Just Accepted}

"Just Accepted" manuscripts have been peer-reviewed and accepted for publication. They are posted online prior to technical editing, formatting for publication and author proofing. The American Chemical Society provides "Just Accepted" as a service to the research community to expedite the dissemination of scientific material as soon as possible after acceptance. "Just Accepted" manuscripts appear in full in PDF format accompanied by an HTML abstract. "Just Accepted" manuscripts have been fully peer reviewed, but should not be considered the official version of record. They are citable by the Digital Object Identifier (DOI®). "Just Accepted" is an optional service offered to authors. Therefore, the "Just Accepted" Web site may not include all articles that will be published in the journal. After a manuscript is technically edited and formatted, it will be removed from the "Just Accepted" Web site and published as an ASAP article. Note that technical editing may introduce minor changes to the manuscript text and/or graphics which could affect content, and all legal disclaimers and ethical guidelines that apply to the journal pertain. ACS cannot be held responsible for errors or consequences arising from the use of information contained in these "Just Accepted" manuscripts. 


\title{
Unhindered Brownian Motion of Individual
} Nanoparticles in Liquid Phase Scanning Transmission Electron Microscopy

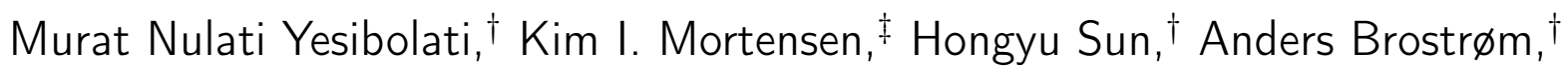 \\ Sofie Tidemand-Lichtenberg, ${ }^{\dagger}$ and Kristian Mølhave*, ${ }^{*}$ \\ †DTU Nanolab, National Centre for Nano Fabrication and Characterization, Technical \\ University of Denmark, DK-2800 Kgs. Lyngby, Denmark \\ $\ddagger D T U$ Health Tech, Department of Health Technology, Technical University of Denmark, \\ DK-2800 Kgs. Lyngby, Denmark \\ E-mail: krmo@dtu.dk
}

\begin{abstract}
Liquid phase transmission electron microscopy (LPTEM) offers label-free imaging of nanoparticle (NP) processes in liquid with sub-nanometer spatial and millisecond temporal resolution. However, LPTEM studies have reported only on NPs moving ordersof-magnitude slower than expected from bulk aqueous liquid conditions, likely due to strong interactions with the LPTEM liquid-enclosing membranes. We demonstrate how scanning transmission electron microscope (STEM) imaging can be used to measure the motion of individual NPs and agglomerates, which are not hindered by such interactions. Only at low electron flux do we find that individual NPs exhibit Brownian motion consistent with optical control experiments and theoretical predictions for unhindered passive diffusive motion in bulk liquids. For increasing electron flux, we find increasingly faster-than-passive motion that still appears effectively Brownian. We discuss the


possible origins of this beam-sample interaction. This establishes conditions for the use of STEM as a reliable tool for imaging nanoscale hydrodynamics in situ TEM.

Keywords:Brownian motion, diffusion, liquid cell, scanning transmission electron microscopy, single particle, low flux

How nanoparticles (NPs) in a liquid suspension grow, move, and interact with each other and surrounding interfaces are of fundamental interest in, e.g., colloidal matter, ${ }^{1,2}$ biomedical applications ${ }^{3}$ microfluidics, ${ }^{4}$ and artificial micro/nanoscopic motors. ${ }^{5,6}$ Traditionally, imaging of such liquid processes has been limited to optical microscopy (OM). Bulk-level methods such as conventional OM and light scattering methods such as dynamic light scattering (DLS) cannot deliver nanometer-spatial-resolution at the single-particle level. Alternatively, optical localization microscopy can track the center positions of fluorescently labeled particles, ${ }^{7,8}$ if they are separated by more than the typically $200 \mathrm{~nm}$ optical resolution limit. In denser samples, various super-resolution microscopy modalities may be used to circumvent this limit on fluorescent samples. ${ }^{8-10}$ Recently, liquid phase transmission electron microscopy (LPTEM) has revolutionized the access to nanoscale, label-free imaging of a wide variety of liquid processes. ${ }^{11,12}$ Typically, the liquid cells used for LPTEM consist of electron-transparent silicon nitride ( $\mathrm{SiNx}$ ) windows suspended on two Si chips, which enclose a liquid sample layer with a thickness ranging from a few hundred nanometers to a couple of microns (Figure 1a, and Supporting Information Note 1). With LPTEM, NP dynamics, e.g., nucleation and growth, ${ }^{13-16}$ self-assembly, ${ }^{17-23}$ and interactions ${ }^{16,17,23-27}$ have been studied with sub-nanometer spatial resolution and millisecond temporal resolution.

If the suspended particles are influenced only by thermal forces caused by the collisions with solvent molecules, with no additional external forces, each particle exhibits unhindered and passive Brownian motion with a diffusion coefficient, $D$, given by the Stokes-Einstein equation (Supporting Information; Methods). In this case, frame-to-frame step displacements of the particle follow a normal distribution and the mean-squared displacement (MSD) in $d$ dimensions, $\mathrm{MSD}=2 d D \Delta t^{\alpha}$, is proportional to the time lag $\Delta t$, i.e. with exponent 
$\alpha=1$.

Numerous LPTEM studies have investigated the motion of NPs, but the NPs chosen for study were likely interacting with the enclosing liquid cell membranes: All previous LPTEM studies ${ }^{15,16,23,28-37}$ have found exceptionally slow diffusive motion of NPs compared to unhindered and passive diffusion. Even when Chee et al. considered only the fastest motion observed, the instantaneous diffusion coefficients were still 20 times smaller than predictions for bulk conditions. ${ }^{32}$ Thus, every diffusion coefficient observed in LPTEM has been 2-9 orders of magnitude smaller than that of unhindered and passive diffusive motion in bulk liquid. In a recent study reporting on highly viscous solutions, $>100$ times that of water, and thus under conditions far from the typically aqueous LPTEM studies, bulk level diffusion was observed using very low flux. ${ }^{38}$

The reported several orders-of-magnitude reduction in diffusion coefficients cannot be explained by a simple increase in the hydrodynamic drag due to thin layer confinement, such as the Faxén correction, see Supporting Information Notes $2-5 .{ }^{28,39,40}$ In all cases, the dynamics had non-normally distributed step displacements, power-law distributed waiting times between flights, and MSDs that scaled with $\Delta t$ as a power-law with $\alpha<1$, which indicate sub-diffusive motion. ${ }^{41}$ Additionally, NPs were influenced by the beam causing them to move in ${ }^{42}$ or out ${ }^{43}$ of the field of view (FOV). Among the suggestions for explanations are inhomogeneous charging of the $\mathrm{SiNx}^{32}$ or a thin liquid layer with high viscosity near the membrane interface, ${ }^{28}$ but the mechanisms behind the varieties of observed slow dynamics are not yet fully understood.

While, slow-moving, surface-interacting NPs are well-suited for high-resolution imaging, the sole focus on such NPs raises a broader concern about the validity of LPTEM results as representative of bulk conditions, ${ }^{44}$ due to the clear presence of a strong interaction that could influence many other experimental aspects than merely motion. Moreover, the lack of observations of unhindered diffusion in water could lead to the false perception that such NP motion does not occur in LPTEM. 
In aqueous LPTEM, currently it is not known: (i) to what extent unhindered Brownian motion is observable, (ii) how beam-irradiation influences the motion of NPs moving unhindered between the two membranes, and (iii) if LPTEM could be appropriate as a high-resolution tracking method for NP dynamics in bulk liquid, free from uncertainties due to membrane interactions. If clarified, these aspects would form a foundation for LPTEM studies of processes with freely-moving NPs and provide a valuable model system for bulk liquid conditions, which could open for a range of new experimental designs.

To address this, we used LPTEM in STEM mode with a high-angle annular dark-field (HAADF) detector to image $150 \mathrm{~nm}$ Au citrate-stabilized NPs in a custom-built liquid cell (Supporting Information Note 1). To minimize influence from membrane surfaces, we imaged within the region with 3-4 $\mathrm{\mu m}$ thick liquid in the middle of the bulging cell. The theoretical values for the diffusion coefficients that we use for comparison below are the bulk values reduced by Faxén's mid-plane surface proximity correction at the thickness estimated for each experiment (Supporting Information Note 3-5). The liquid thickness is beyond the inelastic mean free path, ${ }^{45}$ which leads to STEM contrast inversion from multiple scattering causing the Au particles to appear dark (Figure 1b, Supporting Information Note 6). ${ }^{46,47}$

In order to minimize electron-beam effects on NP dynamics, we used electron fluxes of $0.14-3.5 e^{-} /\left(\AA^{2} \times \mathrm{s}\right)$. These fluxes (Supporting Information Note 6) are low compared to typical values of $1-100 e^{-} /\left(\AA^{2} \times \mathrm{s}\right)$ in the LPTEM literature focusing on NP dynamics. ${ }^{15,16,24,28,32,33}$

In a slow scanning STEM image (Figure 1b), we found that many NPs stuck to the top (1) and bottom membranes (2), as in previous studies. ${ }^{28}$ Slow "sticky" motion was present for NPs (Figure 1b, (3) interacting with a membrane, as earlier reported. ${ }^{15,16,23,28-36}$ Freely moving NPs are not readily observed in a slow-scan STEM image, as they diffuse too fast compared to the acquisition time. Only an occasional NP moving favorably with respect to the raster scan direction of the beam may be observed as a short streak (Figure 1b, (4))

We reduced the number of pixels and the pixel dwell time of each frame, to obtain a 


\section{a}

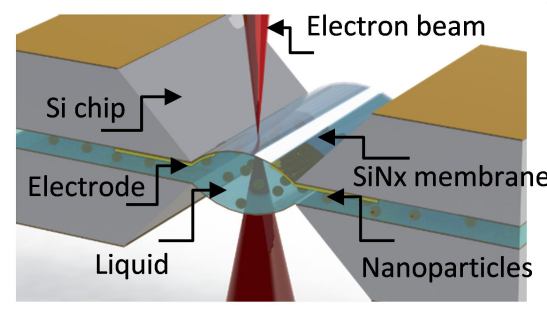

b slow-scan $\Delta t=2 \mathrm{~s}$

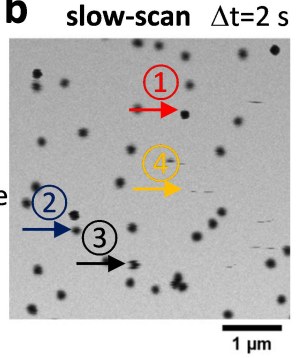

C

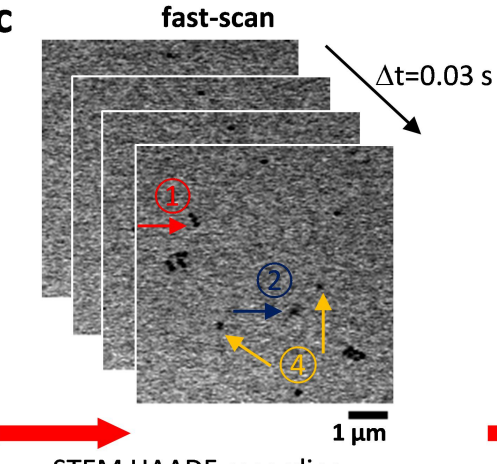

STEM HAADF recording

e

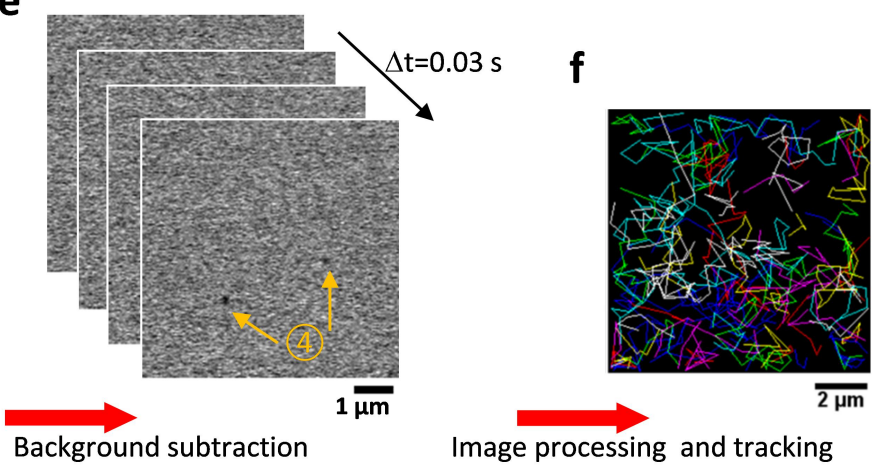

Figure 1: Illustration of the experimental LPTEM STEM setup and analysis. (a) A liquid sample containing Au NPs is sealed between two Si chips with $50 \mathrm{~nm}$ thick electrontransparent SiNx windows in a custom-built liquid TEM holder (Supporting Information Note 1). The windows bulge outward due to the vacuum in the TEM chamber (Supporting Information Notes 2 and 3). (b) Example of slow-scan STEM HAADF image of $150 \mathrm{~nm} \mathrm{Au}$ NPs with an exposure time of $2 \mathrm{~s}$. NPs are either stuck to the top (1) or bottom membrane (2), moving very slowly while interacting with a membrane (3), or moving rapidly between the two membranes, which is seen as a streak only when movement is favorable with respect to the scanning direction of the electron beam (4)). (c,d,e) Sketch of analysis process of fast-scan STEM time-lapse movies (Supporting Information; Methods). NPs moving unhindered (arrows, (4)) are preserved while NPs stuck to a membrane (1) and (2)) are removed by subtraction of a "background" image (temporal average of all frames) from each frame in the time-lapse movie. (f) NPs are detected automatically and the center position of each NP is localized in each frame in the time-lapse movie and thus tracked (Supporting Information; Methods). Typically, each trajectory (colored lines) is clearly separated from others in space and time. 
$33 \mathrm{~Hz}$ frame rate with a large FOV $(10 \mu \mathrm{m} \times 10 \mu \mathrm{m})$ (Supporting Information; Methods). This allowed us to image multiple, individual, freely-diffusing NPs (Figure 1c-e). Our image analysis selected for the freely-moving particles: Particles that were stuck to membranes (or exhibiting orders-of-magnitude slower motion) were excluded from the analysis by subtraction of an all-frames averaged "background" (Figure 1c-e, and Supporting Information; Methods).

The beam broadens as it passes through the liquid but only to the extent that NPs at the top membrane (Figure 1c,d, (1) and the bottom membrane (2)) can be discerned in images by the edge sharpness and contrast. The contrast of freely-moving NPs vary with time within a range between the levels of particles stuck to the top and bottom, indicating varying beam broadening and corresponding z-position. This ensures consistent tracking in the $x y$-plane independent of the $z$-position (Figure 1e, (4)). Due to the noise level, the contrast unfortunately does not allow quantitative tracking of particle motion along the $z$-axis (Supporting Information Note 7).

To characterize the lateral motion of the moving NPs, we tracked the NPs by determining their centroids in each frame of the time-lapse recordings (Figure 1f, Supporting Information; Methods, and Supporting Movies S1-S6). Note that lower electron flux leads to shorter trajectories as tracking is challenging due to the lower signal-to-noise ratio.

We found no significant indication of $(\alpha<1)$ sub- or $(\alpha>1)$ super-diffusion in the measured trajectories for individual NPs, as further discussed and verified below. Thus, it is appropriate to analyze individual NP trajectories as realizations of time-lapse recorded effective Brownian motion specified by a diffusion coefficient and $\alpha=1$. Diffusion coefficients of individual particles (Figure 2a, and Supporting Information Note 8) were determined by the Covariance-based Estimator (CVE, Supporting Information; Methods), ${ }^{48,49}$ which is practically optimal, and superior to traditional analysis based on MSDs (Supporting Information Note 8), especially for shorter trajectories. ${ }^{48-50}$ For each particle at each experimental condition, we found similar diffusion coefficients in the $x$ - and $y$-directions, indicating no influence 
of beam-scan direction (Figure 2a) or significant NP variability in the population. This allowed us to obtain population-averaged diffusion coefficients $\left(\bar{D}_{x, y}\right)$, by averaging the individual estimates of the $2 \mathrm{D}$ diffusion coefficients, using trajectory length as a weight factor (Figure 2b, and Supporting Information; Methods). Note that the CVE analysis implicitly measures and accounts for errors associated with the localization of particles (Supporting Information Note 9).

Since the diffusion coefficients of the individual particles were statistically identical for each experimental condition (Figure 2a), we combined all frame-to-frame displacements of all such particles (Figure 2c). For each experimental condition, the distribution of displacements is favorably described by normal distributions with parameters corresponding to the population-averaged CVE diffusion coefficients $\left(\bar{D}_{x}\right.$ or $\left.\bar{D}_{y}\right)$, as expected if the motion effectively is unhindered Brownian motion. Intriguingly, the variance of the distribution of frame-to-frame displacements of NP trajectories increase with the electron flux (Figure 2c), indicating flux-driven increase of the effective diffusion coefficient. This can also be seen in the population-averaged MSDs (Figure 2d, dots) that show a linear relationship with the lag time within uncertainty - a hallmark of Brownian motion-however, with slopes that increase with increasing electron flux.

Figure 2e shows that only the diffusion coefficient obtained with the lowest electron flux was practically consistent with the value obtained in OM control experiments (Supporting Information; Methods, and Supporting Movie S6). Both these values are close to the theoretical value expected from unhindered and passive Brownian motion (black line, Figure 2e), which also agrees with recent results obtained at very low flux and very high viscosity. ${ }^{38}$ The beam irradiation effect resulting in effective diffusion coefficients exceeding the theoretical value for bulk liquid has not been reported previously. Indeed, flux-related increases in diffusion coefficients have been reported only for NPs interacting with the membranes (such as (3) in Figure 1b), i.e. in a regime where diffusion coefficients remain orders-of-magnitude smaller than the bulk value ${ }^{23,32,35}$ and increases could be caused by reductions in membrane 


\section{a}
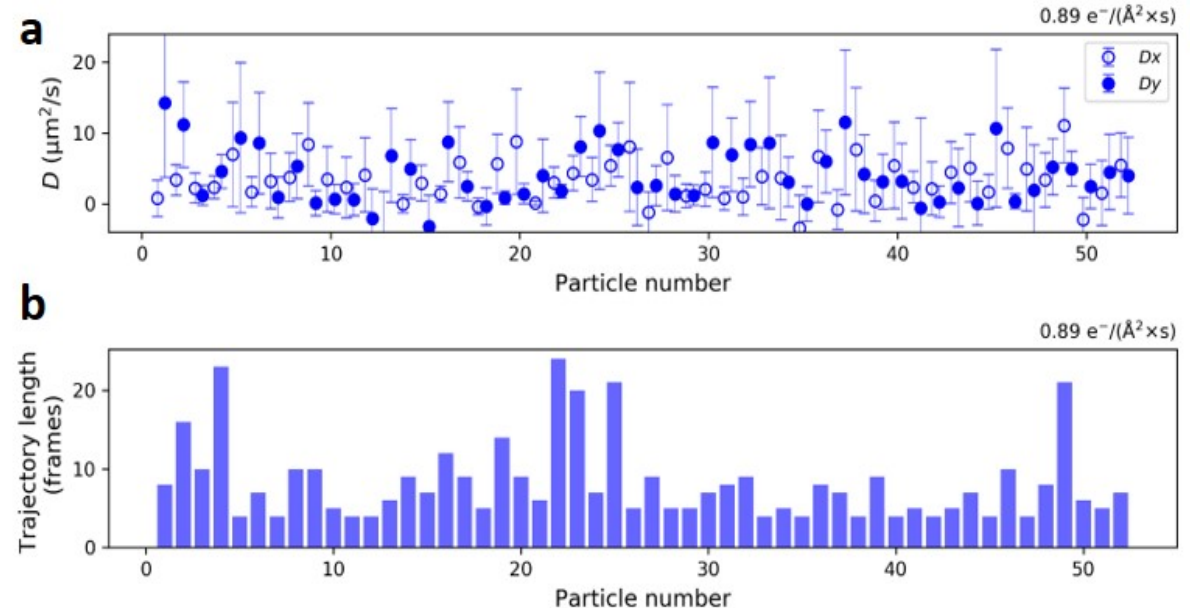

d

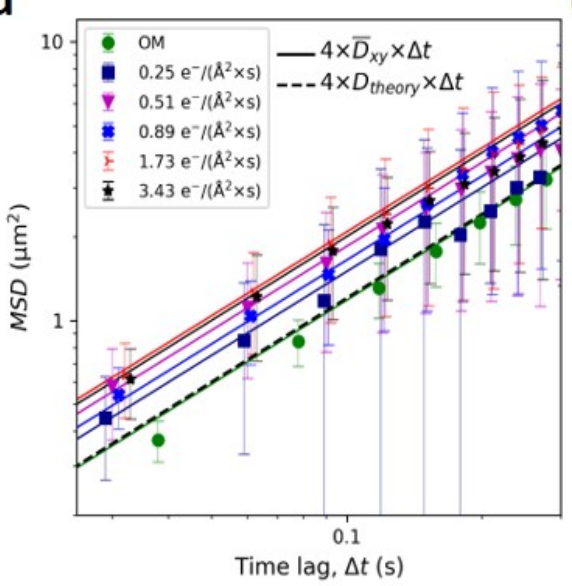

e

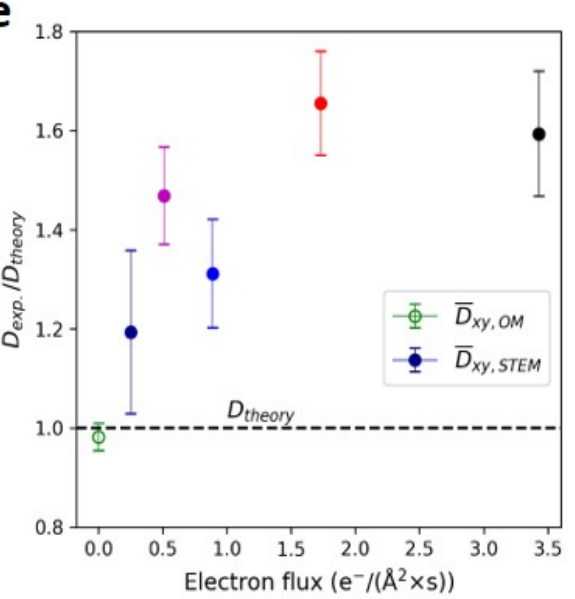

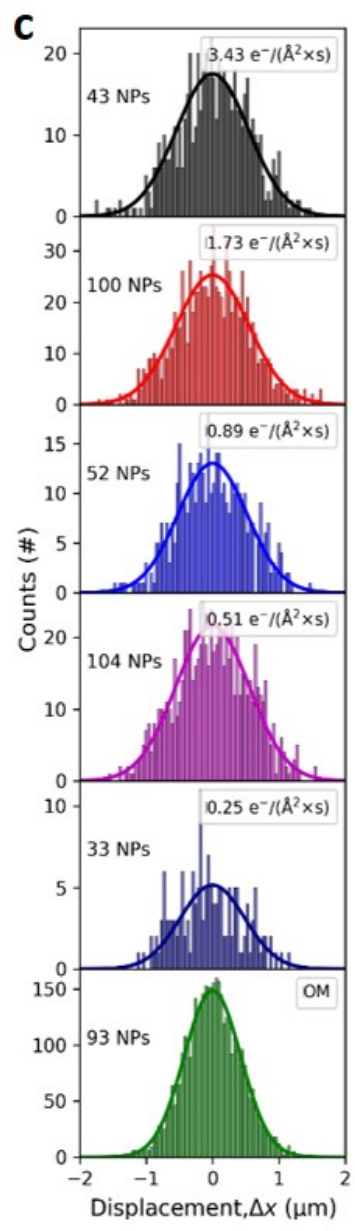

Figure 2: Diffusion analysis of Au NPs in a water solution. (a) Example diffusion coefficients and (b) trajectory lengths of individual Au NPs, here at $0.89 e^{-} /\left(\AA^{2} \times \mathrm{s}\right)$ electron flux. (c) Frame-to-frame $x$-displacement distributions from trajectories of all individual Au NPs under various flux conditions and with OM (see legend, also indicating number of particles). The solid lines indicate normal distributions centered on the origin and with variances corresponding to the population-averaged diffusion coefficients obtained as in (a). Distributions for y-displacements are shown in Supporting Information Note 8. (d) Comparison of the population-averaged conventional MSDs (dots in various colors) to the population-averaged CVE $\bar{D}_{x, y}$ (solid lines in corresponding colors) and the theoretical MSD (dashed line). Error bars indicate SEM and increase with $\Delta t$. (e) Population-averaged diffusion coefficients at different electron fluxes and with OM. $D_{\text {theory }}$ (dashed line) is the theoretical value for unhindered and passive diffusion with Faxén's mid-plane surface proximity correction (Supporting Information Note 5). Error bars indicate the standard error of mean(SEM). The data in (a) and (d) are offset slightly in the horizontal direction to show the error bars more clearly. 
interaction, while in this case there is an additional force at play.

Note that direct determination of diffusion coefficients for individual particles from a fit of a straight line to MSD data is ill-advised (Supporting Information Note 8), due to the fact that the MSD values at different time lags are highly correlated, since they are estimated from the same trajectory. ${ }^{48,50}$ For the same reason, MSD plots of individual trajectories are notoriously difficult to use as rigorous basis for determining presence or absence of anomalous behavior (Figure 2d). While there are no indications of super- or sub-diffusion in the MSD plots, that all show a linear dependence on the lag time within uncertainty, this does not alone guarantee effective Brownian motion: one may occasionally observe linear MSDs when the underlying dynamics are governed by sub-diffusion with a heavy-tailed distribution of waiting times, ${ }^{51}$ but that would be accompanied by a non-normal distribution of displacements, which we did not observe (Figure 2c). This is in contrast to the distinctly non-normal distributions observed in other cases for NPs moving slowly near the membranes. ${ }^{32}$ As a statistically rigorous check for effective Brownian motion, we used a power spectrum analysis of the measured displacements ${ }^{49}$ (Supporting Information Note 8). This showed that the motion indeed is effectively Brownian at all applied electron fluxes, but with effective diffusion coefficients that increase with the electron flux.

We corroborated these observations with a similar set of experiments performed with a viscous $70 \mathrm{wt} \%$ glycerol in water solution. Also in this case, the observed diffusion coefficients increased with the electron flux reaching up to ten times higher diffusion coefficients (Supporting Information Note 10, and Supporting Movie S7).

Though the electron beam has a strong effect on the NP motion, this motion is consistent with effective Brownian motion at all fluxes. The motion does not depend on the beam scanning direction, NP position in FOV, or time of recording in each time-lapse movie (Supporting Information Note 11). A detailed analysis of the particle motion (Supporting Information Note 12) suggests that the diffusion coefficient appears similar on microsecond, millisecond, and second time scales. Hence the increase in diffusion coefficients of NPs is 
not likely due to NPs interacting strongly and locally with the beam, but rather seems to be caused by an interaction present on longer distances and longer times, which results in a force that appears random at the time scale of our observations.

Table 1: Summary of effects that could cause apparent increases in diffusion coefficients. See details in Supporting Information Note 12

\begin{tabular}{|c|c|c|c|}
\hline Possible factors & $\begin{array}{l}s_{\text {water }} \\
{[\mathrm{nm}]}\end{array}$ & \multicolumn{2}{|c|}{$\begin{array}{l}s_{70 \mathrm{wt} \% \text { glycerol }} \\
{[\mathrm{nm}]}\end{array}$} \\
\hline & $\sim 170$ & $\sim 333$ & Experimental observations \\
\hline $\begin{array}{l}\text { Local tempera- } \\
\text { ture increase }\end{array}$ & $\sim 2.6$ & $\sim 10$ & $\begin{array}{l}\text { Temperature measurement and theoretical calculations show that tem- } \\
\text { perature increase during electron beam scanning is less than } 5 \mathrm{~K} \text { in } \\
\text { FOV. }\end{array}$ \\
\hline Hot NP & $\sim 0$ & $\sim 0$ & $\begin{array}{l}\text { The fast-moving NPs are full-size resolved during STEM imaging. } \\
\text { Therefore, the step displacement increase cannot be attributed to the } \\
\text { NP being hot while irradiated. }\end{array}$ \\
\hline Thermophoresis & $\sim 24$ & $\sim 5$ & $\begin{array}{l}\text { Thermal gradients can be large and lead to thermophoretic diffusion, } \\
\text { which is a possible contributing effect. }\end{array}$ \\
\hline $\begin{array}{l}\text { Electrostatic in- } \\
\text { teractions }\end{array}$ & $\sim 0$ & $\sim 0$ & $\begin{array}{l}\text { The electric field from the beam is largely screened by the solu- } \\
\text { tion. Therefore, the Electrophoresis(EP), Dielectrophoresis(DEP), } \\
\text { Electroosmosis(EO), electron beam induced current(EBIC), and po- } \\
\text { tentials from EBIC appear all to be negligible. Any small effect will } \\
\text { also be averaged out as the electron beam scans faster than the NP } \\
\text { diffusion. }\end{array}$ \\
\hline $\begin{array}{l}\text { Momentum } \\
\text { transfer to the } \\
\text { particle }\end{array}$ & $\sim 0$ & $\sim 0$ & Momentum transfer from beam electrons to the NPs is negligible. \\
\hline $\begin{array}{l}\text { Pixel force vor- } \\
\text { tex }\end{array}$ & $\sim 10$ & $\sim 3$ & $\begin{array}{l}\text { A vortex flow can be created in the surrounding liquid that could be } \\
\text { influential }\end{array}$ \\
\hline $\begin{array}{l}\text { FOV force vor- } \\
\text { tex }\end{array}$ & $\sim 0.3$ & $\sim 0.1$ & \\
\hline Diffusiophoresis & & & locity of the order observed. \\
\hline
\end{tabular}

At the maximal value of the diffusion coefficient, the characteristic diffusion displacement length (Figure 2c) between consecutive time-lapse frames at $\Delta t=30 \mathrm{~ms}$ increases above the expected frame-to-frame displacement in bulk condition by about $s_{70 \mathrm{wt} \% \mathrm{glycerol}}=0.3 \mu \mathrm{m}$ in the glycerol solution, and $s_{\text {water }}=0.2 \mu \mathrm{m}$ in water. The interaction thus effectively adds up to $6-11 \mathrm{\mu m} / \mathrm{s}$ random velocity on top of Brownian motion. This could be caused by one or more of a number of effects that we consider in detail in Supporting Information Note 12, discuss below, and summarize in Table 1.

Thermal effects such as radiation induced local temperature increase or "hot" NPs seem 
negligible, which is supported by a microsecond time-scale analysis (Supporting Information Note 12) and direct LPTEM temperature measurements (Supporting Information Note 12). Although absolute temperature increases in the system are too small, thermophoresis from temperature gradients around the scanning beam could be a minor contribution to the increased diffusive motion.

Electrostatic interactions, electrophoresis and electroosmosis are expected to be negligible given the short screening length in the electrolyte and the lack of a microsecond time-scale direct beam interaction (Supporting Information Note 12). Similarly an 'electron wind' effect from beam momentum transfer to NPs or liquid seems a limited contribution.

Among the possible influences, diffusiophoresis, caused by the potential gradients in the electrolyte due to gradients in ion concentrations, ${ }^{52-54}$ such as $\mathrm{pH}$, appears to be a prime candidate capable of increasing the apparent diffusion coefficient. A charge concentration gradient can cause diffusiophoretic motion of the order $5 \mu \mathrm{m} / \mathrm{s}$, for the fastest diffusing radiolytically produced ion $\mathrm{H}^{+}$at about $2 \%$ per micrometer. Stronger $\mathrm{H}^{+}$gradients have been estimated in simulations ${ }^{55}$ for less intense TEM irradiation conditions than the focused STEM beam. A rough estimate gives a diffusiophoretic contribution of the order of the experimentally observed effect (Supporting Information Note 12). Diffusiophoresis is expected to be lower if viscosity is increased (Supporting Information Note 12), contrary to what is observed, but concentration gradients could also be very different. With no validated models for radiolytic pH gradients in STEM, reliable quantitative diffusiophoresis estimates are difficult to make.In addition, the scanning beam further complicates estimating the actual effects in all cases from static condition models.

In LPTEM, most processes of interest involve particles smaller than what we used here to allow direct comparison to OM data. As the particle size decreases, the diffusion coefficient increases, making imaging more challenging. For example, a $20 \mathrm{~nm}$ spherical particle can be imaged in $100 \mathrm{~nm}$ thick water with limited Faxén-correction of about 20\% (Supporting Information Note 4), and a bulk diffusion coefficient of $\mathrm{D}=24 \mathrm{\mu m}^{2} / \mathrm{s}$ at room temperature. 


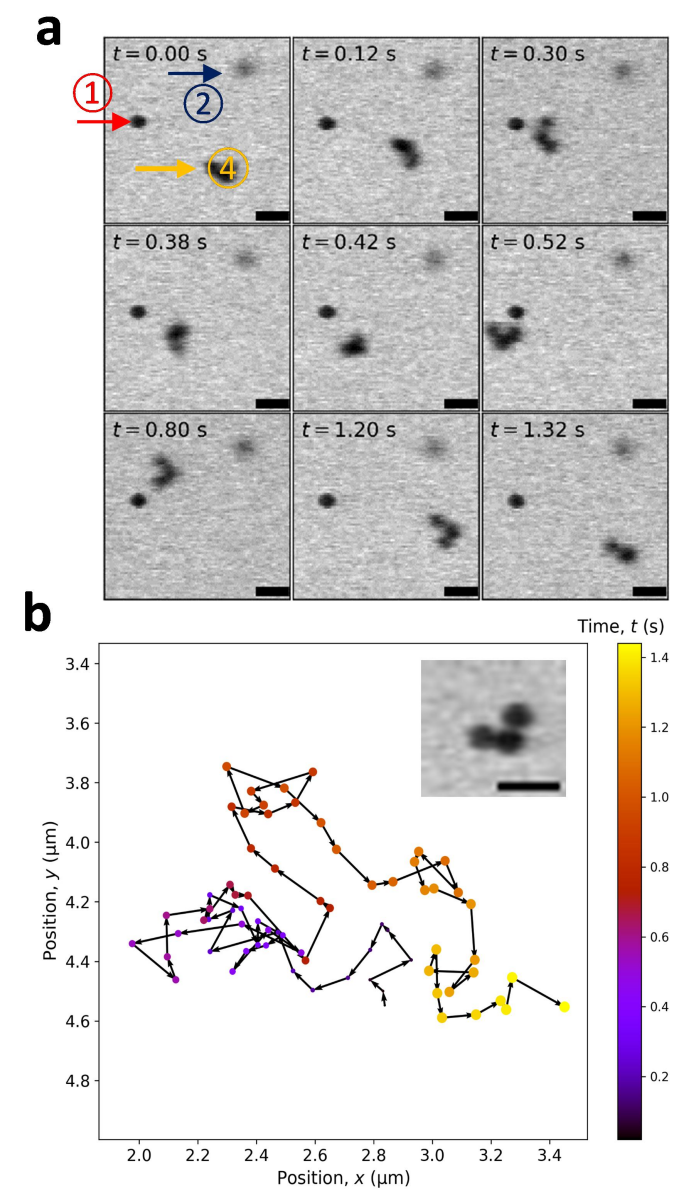

Figure 3: Translational dynamics of a three-particle agglomerate in LPTEM. (a) Timelapse STEM images of a single three-particle agglomerate diffusing and rotating between two SiNx membranes (Supporting Movie S8). The red arrow indicates an Au NP stuck to the top membrane, and the dark-blue arrow one stuck to the bottom membrane. The threeparticle agglomerate (orange arrow) diffuses between the membranes, which can be seen from the contrast and focus variation. (b) The lateral, translational trajectory of the threeparticle agglomerate. The inserted image is a STEM image acquired as the three-particle agglomerate is close to the top membrane showing sharp contours. The solution was $60 \mathrm{wt} \%$ glycerol in water solution, and the electron flux was $4.6 e^{-} /\left(\AA^{2} \times \mathrm{s}\right)$. The scale bar is 300 nm. 

distance per frame, which would be at around 100-1000 frames per second (FPS). This is possible in modern bright field TEM cameras with low electron flux capability, though motion blur may be an issue when the exposure time is not as short as STEM scanning over a single particle. Diffusion can also be deliberately slowed by increasing viscosity, ${ }^{38}$ lowering temperature, or by thin liquid layers as described by Faxén ${ }^{39,40}$ (see Supporting Information Note 5).

Studying motion of complex structures is possible in the present setup, as exemplified by the three-particle agglomerate of NPs in Figure 3 (Supporting Movie 8). With this, e.g., rotational hydrodynamics may be probed, which until now has been reserved to methods with much lower spatial resolution. ${ }^{56-58}$ Such clusters provide better imaging resolution than individual particles, since they diffuse slower due to their size. The three-particle agglomerate was not bound to any membrane, but diffuses and rotates freely between two SiNx membranes. This is indicated by its contrast, which varies between that of particles stuck to the top and bottom membranes. The lateral, translational diffusion coefficient was found to be $0.25 \pm 0.1 \mathrm{\mu m}^{2} / \mathrm{s}$ in a $60 \mathrm{wt} \%$ glycerol solution that has a 10-times higher viscosity than water. This compares favorably to an estimate of $0.2 \mu \mathrm{m}^{2} / \mathrm{s}$, derived from a spheroidal three-particle model that in pure water has $1.95 \mathrm{\mu m}^{2} / \mathrm{ss}^{56}$ While the estimates agree within the uncertainty, a higher experimental value is expected due to the electron flux influence discussed above.

In conclusion, unhindered diffusion has for the first time been observed in LPTEM and quantified with particle tracking. Diffusion coefficients measured at low electron fluxes are consistent with values expected from the Stokes-Einstein equation and OM control experiments at bulk conditions. Optimized LPTEM in STEM mode can hence be used for measuring dynamics of NPs in their native bulk environment at the nanoscale with high spatiotemporal resolution. This opens up for bulk dynamics studies in LPTEM, also e.g. rotation and interactions between particles and substrates. 
Beam-induced faster-than-bulk diffusion was observed with effective diffusion coefficients increasing with the electron flux, still with Gaussian distributions of displacements. The beam influence can be substantial, up to more than a 10 -fold increase for the $70 \%$ glycerol solution. Possible explanations were considered, where contributions were likely caused by thermal gradients leading to thermophoretic diffusion and beam momentum transfer to the liquid by an "electron wind" effect. The main contribution was most likely from diffusiophoresis caused by beam induced concentration gradients of e.g. radiolytic $\mathrm{H}^{+}$.

\section{Acknowledgement}

The authors acknowledge core facilities support from DTU Nanolab; Engineering support from P. T. Jonassen, W. Huyzer and A. Fuller. The authors acknowledge helpful discussions with T. Kasama, U. Mirsaidov, R. Dunin-Borkowski, J.B.Wagner, S. Kadkhodazadeh, M. Beleggia, K. Kroy, F. Cichos, A. Abulaitijiang, H. Bruus and S. Canepa. The research was supported by the Technical University of Denmark, by the Danish Research Council for Technology and Production (Case No. 12-126194), in part by the Advanced Materials for Energy-Water Systems (AMEWS) Center, an Energy Frontier Research Center funded by the U.S. Department of Energy, Office of Science, Basic Energy Sciences, and in part by the VILLUM foundation with project number 00028273. The first author acknowledges the Otto Mønsteds Fond for financially supporting conference participation.

\section{Supporting Information Available}

Supporting Information (.pdf) includes Methods and materials, Supporting Information Notes 1 to 12, Figures S1 to S21, Tables S1 to S3, Legends for Supporting Movies S1 to S8, and References detailing the custom-made liquid cell, liquid thickness estimation under OM, STEM data at different electron flux and extended analysis, and discussion. 
Supporting Movie(.avi) includes 8 trajectory-overlay example movies from various electron fluxes and optical measurements in different liquid solutions.

\section{Conflicts of interest}

There are no conflicts to declare.

\section{Author Contributions}

M.N.Yesibolati, H.Y.Sun, and K.Mølhave proposed and directed the research; M.N.Yesibolati and H.Y.Sun conducted experiments. M.N.Yesibolati, K.I.Mortensen, and K.Mølhave carried out data analysis and wrote the manuscript; A.Brostrøm and S.Tidemand-Lichtenberg assisted with the data analysis. All authors discussed the results and approved the final version of the manuscript.

\section{References}

(1) Manoharan, V. N. Colloidal matter: Packing, geometry, and entropy. Science 2015, $349,1253751$.

(2) Turiv, T.; Lazo, I.; Brodin, A.; Lev, B. I.; Reiffenrath, V.; Nazarenko, V. G.; Lavrentovich, O. D. Effect of Collective Molecular Reorientations on Brownian Motion of Colloids in Nematic Liquid Crystal. Science 2013, 342, 1351-1354.

(3) Nykypanchuk, D.; Strey, H. H.; Hoagland, D. A. Brownian motion of DNA confined within a two-dimensional array. Science 2002, 297, 987-990.

(4) Krishnan, M.; Mojarad, N.; Kukura, P.; Sandoghdar, V. Geometry-induced electrostatic trapping of nanometric objects in a fluid. Nature 2010, 467, 692-U75. 
(5) Astumian, R. D. Thermodynamics and kinetics of a Brownian motor. Science 1997, 276, 917-922.

(6) Hanggi, P.; Marchesoni, F. Artificial Brownian motors: Controlling transport on the nanoscale. Reviews of Modern Physics 2009, 81, 387-442.

(7) Deschout, H.; Zanacchi, F. C.; Mlodzianoski, M.; Diaspro, A.; Bewersdorf, J.; Hess, S. T.; Braeckmans, K. Precisely and accurately localizing single emitters in fluorescence microscopy. Nature Methods 2014, 11, 253-266.

(8) Kempter, S.; Khmelinskaia, A.; Strauss, M. T.; Schwille, P.; Jungmann, R.; Liedl, T.; Bae, W. Single Particle Tracking and Super-Resolution Imaging of Membrane-Assisted Stop-and-Go Diffusion and Lattice Assembly of DNA Origami. ACS Nano 2019, 13, 996-1002.

(9) Rust, M. J.; Bates, M.; Zhuang, X. W. Sub-diffraction-limit imaging by stochastic optical reconstruction microscopy (STORM). Nature Methods 2006, 3, 793-795.

(10) Hess, S. T.; Girirajan, T. P. K.; Mason, M. D. Ultra-high resolution imaging by fluorescence photoactivation localization microscopy. Biophysical Journal 2006, 91, 42584272.

(11) Ross, F. M. Opportunities and challenges in liquid cell electron microscopy. Science 350 , aaa9886.

(12) de Jonge, N.; Ross, F. M. Electron microscopy of specimens in liquid. Nature Nanotechnology 2011, 6, 695-704.

(13) Loh, N. D.; Sen, S.; Bosman, M.; Tan, S. F.; Zhong, J.; Nijhuis, C. A.; Kral, P.; Matsudaira, P.; Mirsaidov, U. Multistep nucleation of nanocrystals in aqueous solution. Nature Chemistry 2017, 9, 77-82. 
(14) Zheng, H. M.; Smith, R. K.; Jun, Y. W.; Kisielowski, C.; Dahmen, U.; Alivisatos, A. P. Observation of Single Colloidal Platinum Nanocrystal Growth Trajectories. Science 2009, 324, 1309-1312.

(15) Woehl, T. J.; Park, C.; Evans, J. E.; Arslan, I.; Ristenpart, W. D.; Browning, N. D. Direct Observation of Aggregative Nanoparticle Growth: Kinetic Modeling of the Size Distribution and Growth Rate. Nano Letters 2014, 14, 373-378.

(16) Lee, J.; Nakouzi, E.; Song, M.; Wang, B.; Chun, J.; Li, D. Mechanistic Understanding of the Growth Kinetics and Dynamics of Nanoparticle Superlattices by Coupling Interparticle Forces from Real-Time Measurements. ACS Nano 2018, 12, 12778-12787.

(17) Tan, S. F.; Chee, S. W.; Lin, G. H.; Mirsaidov, U. Direct Observation of Interactions between Nanoparticles and Nanoparticle Self-Assembly in Solution. Accounts of Chemical Research 2017, 50, 1303-1312.

(18) Lin, G. H.; Chee, S. W.; Raj, S.; Kral, P.; Mirsaidov, U. Linker-Mediated Self-Assembly Dynamics of Charged Nanoparticles. ACS Nano 2016, 10, 7443-7450.

(19) Plamper, F. A.; Gelissen, A. P.; Timper, J.; Wolf, A.; Zezin, A. B.; Richtering, W.; Tenhu, H.; Simon, U.; Mayer, J.; Borisov, O. V.; Pergushov, D. V. Spontaneous Assembly of Miktoarm Stars into Vesicular Interpolyelectrolyte Complexes. Macromolecular Rapid Communications 2013, 34, 855-860.

(20) Kim, B. H.; Heo, J.; Lee, W. C.; Park, J. Liquid-cell Transmission Electron Microscopy for Tracking Self-assembly of Nanoparticles. Jove-Journal of Visualized Experiments $\mathbf{2 0 1 7 , ~ 1 2 8 , 5 6 3 3 5 . ~}$

(21) Lee, W. C.; Kim, B. H.; Choi, S.; Takeuchi, S.; Park, J. Liquid Cell Electron Microscopy of Nanoparticle Self-Assembly Driven by Solvent Drying. Journal of Physical Chemistry Letters 2017, 8, 647-654. 
(22) Liu, Y.; Lin, X.-M.; Sun, Y.; Rajh, T. In Situ Visualization of Self-Assembly of Charged Gold Nanoparticles. Journal of the American Chemical Society 2013, 135, 3764-3767.

(23) Woehl, T. J.; Prozorov, T. The Mechanisms for Nanoparticle Surface Diffusion and Chain Self-Assembly Determined from Real-Time Nanoscale Kinetics in Liquid. The Journal of Physical Chemistry C 2015, 119, 21261-21269.

(24) Tan, S. F.; Raj, S.; Bisht, G.; Annadata, H. V.; Nijhuis, C. A.; Král, P.; Mirsaidov, U. Nanoparticle Interactions Guided by Shape-Dependent Hydrophobic Forces. Advanced Materials (Weinheim, Germany) 2018, 30, 1707077.

(25) Powers, A. S.; Liao, H.-G.; Raja, S. N.; Bronstein, N. D.; Alivisatos, A. P.; Zheng, H. Tracking Nanoparticle Diffusion and Interaction during Self-Assembly in a Liquid Cell. Nano Letters 2017, 17, 15-20.

(26) Liao, H.-G.; Niu, K.-Y.; Zheng, H. In Liquid Cell Electron Microscopy; Ross, F. M., Ed.; Advances in Microscopy and Microanalysis; Cambridge University Press: Cambridge, 2016; pp 191-209.

(27) Chen, Q.; Cho, H.; Manthiram, K.; Yoshida, M.; Ye, X.; Alivisatos, A. P. Interaction Potentials of Anisotropic Nanocrystals from the Trajectory Sampling of Particle Motion using in Situ Liquid Phase Transmission Electron Microscopy. Acs Central Science 2015, 1, 33-39.

(28) Verch, A.; Pfaff, M.; de Jonge, N. Exceptionally Slow Movement of Gold Nanoparticles at a Solid/Liquid Interface Investigated by Scanning Transmission Electron Microscopy. Langmuir 2015, 31, 6956-6964.

(29) Yuk, J. M.; Park, J.; Ercius, P.; Kim, K.; Hellebusch, D. J.; Crommie, M. F.; Lee, J. Y.; Zettl, A.; Alivisatos, A. P. High-Resolution EM of Colloidal Nanocrystal Growth Using Graphene Liquid Cells. Science 2012, 336, 61-64. 
(30) Ring, E. A.; de Jonge, N. Video-frequency scanning transmission electron microscopy of moving gold nanoparticles in liquid. Micron 2012, 43, 1078-1084.

(31) Zheng, H. M.; Claridge, S. A.; Minor, A. M.; Alivisatos, A. P.; Dahmen, U. Nanocrystal Diffusion in a Liquid Thin Film Observed by in Situ Transmission Electron Microscopy. Nano Letters 2009, 9, 2460-2465.

(32) Chee, S. W.; Baraissov, Z.; Loh, N. D.; Matsudaira, P. T.; Mirsaidov, U. DesorptionMediated Motion of Nanoparticles at the Liquid-Solid Interface. Journal of Physical Chemistry C 2016, 120, 20462-20470.

(33) Cepeda-Pérez, E.; de Jonge, N. Dynamics of gold nanoparticle clusters observed with liquid-phase electron microscopy. Micron 2019, 117, 68-75.

(34) Lu, J.; Aabdin, Z.; Loh, N. D.; Bhattacharya, D.; Mirsaidov, U. Nanoparticle Dynamics in a Nanodroplet. Nano Letters 2014, 14, 2111-2115.

(35) Chee, S. W.; Anand, U.; Bisht, G.; Tan, S. F.; Mirsaidov, U. Direct Observations of the Rotation and Translation of Anisotropic Nanoparticles Adsorbed at a Liquid-Solid Interface. Nano Letters 2019, 19, 2871-2878.

(36) Fu, X. W.; Chen, B.; Tang, J.; Zewail, A. H. Photoinduced nanobubble-driven superfast diffusion of nanoparticles imaged by 4D electron microscopy. Science Advances 2017, 3, e1701160.

(37) Ren, A.; Lu, D.; Wong, E.; Hauwiller, M. R.; Alivisatos, A. P.; Ren, G. Real-time observation of dynamic structure of liquid-vapor interface at nanometer resolution in electron irradiated sodium chloride crystals. Scientific Reports 2020, 10, 8596.

(38) Welling, T. A. J.; Sadighikia, S.; Watanabe, K.; Grau-Carbonell, A.; Bransen, M.; Nagao, D.; van Blaaderen, A.; van Huis, M. A. Observation of Undamped 3D Brownian 
Motion of Nanoparticles Using Liquid-Cell Scanning Transmission Electron Microscopy. Particle E Particle Systems Characterization 2020, 37, 2000003.

(39) Faxen, H. Kleine Mitteilungen. ZAMM - Journal of Applied Mathematics and Mechanics / Zeitschrift für Angewandte Mathematik und Mechanik 1927, 7, 79-81.

(40) Faxen, H. Influence of walls on the drag of a small sphere moving in a viscous fluid (In German). PhD. Thesis, 1921.

(41) Metzler, R.; Klafter, J. The random walk's guide to anomalous diffusion: a fractional dynamics approach. Physics Reports 2000, 339, 1-77.

(42) Zheng, H.; Mirsaidov, U. M.; Wang, L.-W.; Matsudaira, P. Electron Beam Manipulation of Nanoparticles. Nano Letters 2012, 12, 5644-5648.

(43) White, E. R.; Mecklenburg, M.; Shevitski, B.; Singer, S. B.; Regan, B. C. Charged nanoparticle dynamics in water induced by scanning transmission electron microscopy. Langmuir: the ACS journal of surfaces and colloids 2012, 28, 3695-3698.

(44) Parent, L. R.; Bakalis, E.; Proetto, M.; Li, Y.; Park, C.; Zerbetto, F.; Gianneschi, N. C. Tackling the Challenges of Dynamic Experiments Using Liquid-Cell Transmission Electron Microscopy. Accounts of Chemical Research 2018, 51, 3-11.

(45) Yesibolati, M. N.; Lagana, S.; Kadkhodazadeh, S.; Mikkelsen, E. K.; Sun, H.; Hansen, O.; Zaluzec, N. J.; Mølhave, K. Electron Inelastic Mean Free Path in Water. Nanoscale 2020, DOI: 10.1039/D0NR04352D.

(46) Gnanasekaran, K.; de With, G.; Friedrich, H. Quantification and optimization of ADFSTEM image contrast for beam-sensitive materials. Royal Society open science $\mathbf{2 0 1 8}$, $5,171838-171838$.

(47) Van den Broek, W.; Rosenauer, A.; Goris, B.; Martinez, G. T.; Bals, S.; Van Aert, S.; 
Van Dyck, D. Correction of non-linear thickness effects in HAADF STEM electron tomography. Ultramicroscopy 2012, 116, 8-12.

(48) Vestergaard, C. L.; Blainey, P. C.; Flyvbjerg, H. Optimal estimation of diffusion coefficients from single-particle trajectories. Physical Review E 2014, 89.

(49) Vestergaard, C. L.; Pedersen, J. N.; Mortensen, K. I.; Flyvbjerg, H. Estimation of motility parameters from trajectory data. European Physical Journal-Special Topics 2015, 224, 1151-1168.

(50) Berglund, A. J. Statistics of camera-based single-particle tracking. Physical Review E 2010, 82.

(51) Lubelski, A.; Sokolov, I. M.; Klafter, J. Nonergodicity Mimics Inhomogeneity in Single Particle Tracking. Physical Review Letters 2008, 100, 250602.

(52) Prieve, D. C.; Malone, S. M.; Khair, A. S.; Stout, R. F.; Kanj, M. Y. Diffusiophoresis of charged colloidal particles in the limit of very high salinity. Proceedings of the National Academy of Sciences of the United States of America 2019, 116, 18257-18262.

(53) Shin, S.; Um, E.; Sabass, B.; Ault, J. T.; Rahimi, M.; Warren, P. B.; Stone, H. A. Size-dependent control of colloid transport via solute gradients in dead-end channels. Proceedings of the National Academy of Sciences of the United States of America 2016, $113,257-261$.

(54) Velegol, D.; Garg, A.; Guha, R.; Kar, A.; Kumar, M. Origins of concentration gradients for diffusiophoresis. Soft Matter 2016, 12, 4686-4703.

(55) Schneider, N. M.; Norton, M. M.; Mendel, B. J.; Grogan, J. M.; Ross, F. M.; Bau, H. H. Electron-Water Interactions and Implications for Liquid Cell Electron Microscopy. The Journal of Physical Chemistry C 2014, 118, 22373-22382. 
(56) Hoffmann, M.; Wagner, C. S.; Harnau, L.; Wittemann, A. 3D Brownian Diffusion of Submicron-Sized Particle Clusters. ACS Nano 2009, 3, 3326-3334.

(57) Hunter, G. L.; Edmond, K. V.; Elsesser, M. T.; Weeks, E. R. Tracking rotational diffusion of colloidal clusters. Opt. Express 2011, 19, 17189-17202.

(58) Fung, J.; Martin, K. E.; Perry, R. W.; Kaz, D. M.; McGorty, R.; Manoharan, V. N. Measuring translational, rotational, and vibrational dynamics in colloids with digital holographic microscopy. Opt. Express 2003, 19, 8051-8065. 


\section{Graphical TOC Entry}

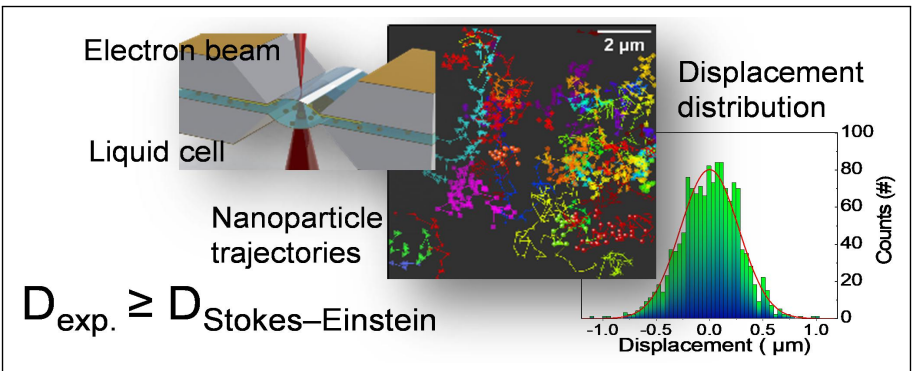

